\title{
Objeto virtual de aprendizagem sobre rastreamento do câncer do colo do útero
}

Cervical cancer tracking virtual learning object Objeto virtual de aprendizaje sobre rastreo de cáncer de cuello uterino

Marcelo de Souza Dutra Davilla ${ }^{1}$ io https://orcid.org/0000-0003-2996-8608

Cândida Caniçali Primo ${ }^{1}$ id https://orcid.org/0000-0001-5141-2898

Márcia Valéria de Souza Almeida ${ }^{1}$ id https://orcid.org/0000-0002-1318-7084

Franciéle Marabotti Costa Leite ${ }^{1}$ io https://orcid.org/0000-0002-6171-6972

Hugo Cristo Sant'Anna ${ }^{1}$ io https://orcid.org/0000-0003-4890-6728

Rodrigo Jensen ${ }^{1}$ id https://orcid.org/0000-0001-6191-2001

Eliane de Fátima Almeida Lima ${ }^{1}$ io https://orcid.org/0000-0001-5128-3715

Como citar: Davilla MS, Primo CC, Almeida MV, Leite FM, Sant'Anna HC, Jensen R. Objeto virtual de aprendizagem sobre rastreamento do câncer do colo do útero. Acta Paul Enferm. 2021;34:AAPE00063.

DOI

http://dx.doi.org/10.37689/actaape/2021A000063

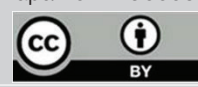

Educacão em enfermagem: Sain Neoplasias do colo do útero; Tecnologia educacional; Hipermidia

Keywords

Education, nursing; Women's health; Uterine cervical neoplasms; Education technology:

Hypermedia

Descriptores

Educación en Enfermería; Salud de la Mujer: Neoplasias del cuello uterino; Tecnología educacional; Hipermedia

Submetido 21 de Janeiro de 2020

Aceito

23 de Setembro de 2020

Autor correspondente

Cândida Caniçali Primo

E-mail: candida.primo@ufes.br

\section{Resumo}

Objetivo: Descrever o desenvolvimento e avaliação do conteúdo de um objeto virtual de aprendizagem sobre prevenção e rastreamento do câncer do colo do útero.

Métodos: Pesquisa aplicada de desenvolvimento tecnológico realizada em três etapas: elaboração do conteúdo técnico científico; avaliação do conteúdo teórico com 21 enfermeiros por meio de Painel Delphi online e construção do objeto virtual de aprendizagem.

Resultados: 0 objeto virtual de aprendizagem possui 7 módulos, 65 telas de conteúdo, com carga horária total de 60 horas. A avaliação do conteúdo apresentou concordância acima de 0,80, sendo considerado adequado.

Conclusão: Essa tecnologia é uma alternativa motivadora de educação em saúde, capaz de otimizar a difusão da informação sobre o manuseio e coleta do preventivo, melhorando a qualidade da assistência e a prevenção do câncer do colo do útero.

\section{Abstract}

Objective: To describe the content development and assessment of a virtual learning object on cervical cancer prevention and tracking.

Methods: Applied technological development research carried out in three stages: scientific content elaboration; theoretical content assessment with 21 nurses through online Delphi Panel and virtual learning object construction.

Results: The virtual learning object has 7 modules, 65 content screens, with a total workload of 60 hours. The content assessment showed an agreement above 0.80 , being considered adequate.

Conclusion: This technology is a motivating alternative for health education, capable of optimizing information dissemination on the handling and collection of the preventive, improving the quality of care and cervical cancer prevention.

\section{Resumen}

Objetivo: Describir el desarrollo y evaluación de contenido de un objeto virtual de aprendizaje sobre prevención y rastreo de cáncer de cuello uterino.

Métodos: Estudio aplicado de desarrollo tecnológico realizado en tres etapas: elaboración del contenido técnico científico, evaluación del contenido teórico por 21 enfermeros mediante el Panel Delphi en línea y construcción del objeto virtual de aprendizaje. 
Resultados: El objeto virtual de aprendizaje posee 7 módulos, 65 pantallas de contenido, con una carga horaria total de 60 horas. La evaluación del contenido presentó concordancia superior a 0,80 y fue considerado adecuado.

Conclusión: Esta tecnología es una alternativa motivadora de educación en salud, que tiene la capacidad de optimizar la difusión de la información sobre la toma de muestra de Papanicolau y su manipulación y mejorar la calidad de la atención y prevención de cáncer de cuello uterino.

\section{Introdução}

O câncer é um problema de saúde pública, especialmente entre os países em desenvolvimento. Em relação ao câncer do colo do útero no biênio 20182019, no Brasil, estimam-se 16.370 casos novos para cada ano, com um risco estimado de 15,43 casos a cada 100 mil mulheres, ocupando a terceira posição no ranking das estimativas de novos casos de câncer para o ano de 2018, segundo sexo e localização primária. ${ }^{(1)}$

A detecção precoce do câncer do colo do útero, por meio do exame citopatológico (teste do Papanicolau), para identificar lesôes precursoras e diagnosticar a doença precocemente leva a diminuição dos índices de morbimortalidade, pois o diagnóstico na fase inicial possui alto índice de cura. (2) O êxito no rastreamento do câncer do colo do útero e de suas lesóes precursoras depende da acuidade diagnóstica do exame, sendo imprescindível a qualidade da assistência na coleta do teste, bem como a capacitação e a atualização do profissional em relação aos métodos e protocolos atuais. Nessa perspectiva, as tecnologias educacionais digitais estão sendo cada vez mais utilizadas como estratégias de educação continuada ou permanente para o aprimoramento técnico dos profissionais de saúde. ${ }^{(3-5)}$

As tecnologias educacionais abrangem os conhecimentos científicos obtidos através de um planejamento, controle, produção e execução num conjunto sistemático que envolve todo o processo educacional formal e informal entre o educador e o educando. ${ }^{(6)}$ Tem se uma diversidade de tecnologias como jogos, aplicativos, hipertexto, hipermídias, manequim simulador de alta fidelidade, simulador em ambiente virtual, vídeos, cursos completos, objeto virtual de aprendizagem (OVA), sites, chats, blogs, fóruns, teleconferência e webconferência. A tecnologia digital permite um estudo de forma independente, flexível, desenvolve distintas habilidades, contribui na autonomia do aprendizado, na as- sociação da teoria com a prática, e na aprendizagem significativa. $^{(4,5)}$

O OVA é um recurso tecnológico com suporte multimídia e linguagem hipermídia reutilizável para aprendizagem interativa mediante material educativo com conteúdos didáticos e animaçóes, permeados pela interdisciplinaridade, interatividade, além de complementos e exercícios. ${ }^{(7)}$ Hipermídias são o conjunto de várias mídias computadorizadas de informaçôes com texto, som, gráficos e/ou vídeo, interconectadas por encadeamentos lógicos náo lineares que permitem que os usuários sigam ótimos caminhos através do material e também dos sistemas usados para criar e exibir esta informação. ${ }^{(8-10)}$

O OVA oferece as vantagens de poder ser realizado a distância, ter flexibilidade e possibilidade do estudante administrar o seu local e horário de estudo de acordo com suas necessidades e favorece o acesso independente das características regionais de centros urbanos ou lugares remotos. ${ }^{(4,5,7,9,10)}$ Frente a essas questôes, questiona-se quais conteúdos devem conter um OVA sobre prevenção e rastreamento do câncer do colo do útero? Assim, este estudo tem como objetivo descrever o desenvolvimento e avaliação do conteúdo de um objeto virtual de aprendizagem sobre prevenção e rastreamento do câncer do colo do útero.

\section{Métodos}

Trata-se de pesquisa aplicada ${ }^{(11)}$ de desenvolvimento tecnológico realizada em três etapas: 1) elaboração do conteúdo teórico; 2) avaliação do conteúdo teórico; e 3) construção do OVA.

Para a elaboração do conteúdo do OVA foram utilizados os manuais do Ministério da Saúde (Caderno de Atenção Básica no 13: controle dos cânceres do colo do útero e da mama; Estimativa 2018: incidência de câncer no Brasil, Dados dos registros de base populacional - Vol. IV; Ações de en- 
fermagem para o controle do câncer: uma proposta de integração ensino-serviço e a Nomenclatura brasileira para laudos cervicais e condutas preconizadas: recomendações para profissionais de saúde); as diretrizes da Sociedade Brasileira de Ginecologia e Obstetrícia; e livros textos de ginecologia e de enfermagem.

Foram também elaboradas atividades de verificação de aprendizagem, compostas por perguntas ou estudos de caso com questóes de múltipla escolha. Essas atividades foram corrigidas por profissional de pedagogia. O conteúdo foi revisado e corrigido por uma equipe de professores de letras/português. $\mathrm{O}$ conteúdo foi elaborado no período de março a julho de 2018. Toda a etapa de elaboração e ilustração da tecnologia educativa foi feita com a colaboração de uma equipe de design.

A avaliação de conteúdo foi realizada por juízes, por meio de painel Delphi online, no período de agosto a outubro de 2018 . Na literatura científica não há um padrão estabelecido em relação aos critérios para a definição da quantidade e características dos juízes. Dessa forma, destaca-se a importância da seleção de profissionais de saúde que possuam experiência clínica e conhecimento teórico no assunto. ${ }^{(12)}$

O grupo de juízes foi formado por enfermeiros que atuavam na atenção primária à saúde, professores e enfermeiros com experiência de, no mínimo, dois anos em uma das áreas de interesse: Saúde da Mulher, Enfermagem Obstétrica ou Ginecológica. Os juízes foram selecionados por meio da rede de contatos dos pesquisadores pela técnica de amostragem snowball ou "bola de neve", tratando-se, portanto, de uma amostragem por conveniência. Os juízes receberam por meio de correio eletrônico uma carta-convite com informaçôes a respeito do estudo. Ao aceitar, o juiz assinava o Termo de Consentimento Livre e Esclarecido e recebia um instrumento virtual no Google Formulário.

No instrumento de avaliação organizado por conteúdo/telas, o juiz poderia assinalar uma das opçôes: "Adequado", "Parcialmente adequado" e "Inadequado" para cada tela. Adotou-se o índice de $80 \%$ como nível mínimo de consenso. As situações que apresentassem concordância inferior deveriam ser reformuladas, acatadas as sugestóes e reenviado para os juízes, para nova avaliação até o alcance de $80 \%$. $^{(12)}$

$\mathrm{Na}$ terceira etapa, o OVA foi elaborado utilizando a plataforma Fred, desenvolvida pelo Laboratório e Observatório de Ontologias Projetuais (Loop) da Universidade Federal do Espírito Santo (Ufes), no período de março a junho de 2019. Esta plataforma implementa sistemas personalizados de instrução (SPIs), conforme definidos por Keller ${ }^{(13)}$ e potencializados pelo avanço do ensino a distância via internet na última década. ${ }^{(14)}$ Os princípios de SPIs ${ }^{(15)}$ presentes na plataforma orientaram a concepção do curso por meio de personalizaçóes de objetivos instrucionais, do ambiente das tarefas e atividades do OVA, do nível de mediação da instrução pela plataforma, formas de avaliação e reflexóes do aprendiz sobre seus progressos.

O estudo foi aprovado no Comitê de Ética em Pesquisa do Centro de Ciências da Saúde da Universidade Federal do Espírito Santo, sob número CAAE 57930016.0.0000.5060.

\section{Resultados}

Para a elaboração do conteúdo do OVA foram utilizadas as diretrizes nacionais do Ministério da Saúde e das sociedades tendo em vista que são baseadas nas melhores evidências e boas práticas. A arquitetura de conteúdo do OVA foi organizado em sete módulos, sendo o primeiro de apresentação com o pré-teste, cinco módulos com conteúdos teóricos e o último com o pós-teste e emissão de certificado. $\mathrm{O}$ objetivo deste OVA é ser uma ferramenta complementar no treinamento e capacitação de enfermeiros/profissionais de saúde acerca da prevençâo e rastreamento ou screening do câncer do colo do útero.

Uma atividade de verificação de aprendizagem, composta por perguntas ou estudos de caso que contemplam questóes de múltipla escolha encontra-se ao final de cada tela. No total foram elaboradas 53 questóes distribuídas no decorrer do OVA. As telas empregam ilustraçóes, fotos, infografias e vídeos conforme a natureza do conteúdo.

O OVA possui carga horária total de 60 horas. $\mathrm{O}$ usuário pode cursar os módulos de forma indepen- 
dente ou sequenciada e repetir os módulos quantas vezes for necessário. $\mathrm{O}$ módulo de apresentação e finalização do OVA contém 26 questôes de pré-teste e pós-teste, sendo as mesmas questóes aplicadas no início e ao final, para avaliar a assimilação do conteúdo proposto pela hipermídia, a partir do aumento de acertos nas questóes. Ao final é emitido automaticamente um certificado com a carga horária total.

Na etapa de avaliaçáo, foram convidados 30 juízes 21 responderam ao e-mail. Os juízes apresentavam o seguinte perfil: 12 (60\%) tinham mais 40 anos, sen- do que 16 (86\%) eram do sexo feminino e, todos tinham, no mínimo, nove anos de formação acadêmica em uma das áreas de interesse: Saúde da Mulher (13,5\%), Enfermagem Obstétrica ou Ginecológica $(27,1 \%)$ ou na Atenção Primária à Saúde $(59,4 \%)$.

Por meio do instrumento, os juízes avaliaram o conteúdo de cada módulo organizado em telas. Todos as telas dos módulos de conteúdo foram avaliadas na primeira rodada com mais de $90 \%$ de concordância entre os 21 enfermeiros juízes, não sendo necessário mais rodadas, conforme descrito no quadro 1 .

Quadro 1. Estrutura do conteúdo do Objetivo Virtual de Aprendizagem e Índice de Validade de Conteúdo

\begin{tabular}{|c|c|c|c|}
\hline Módulos & Telas & Conteúdo & IVC \\
\hline \multirow{10}{*}{$\begin{array}{l}\text { Módulo I } \\
0 \text { que é Câncer }\end{array}$} & Tela 1 & Tecnologia educacional para atualização do rastreamento do câncer do colo do útero & 100,0 \\
\hline & Tela 2 & Conteúdo programático do OVA & 100,0 \\
\hline & Tela 3 & Tela de apresentação & 100,0 \\
\hline & Tela 4 & Tela de créditos & 100,0 \\
\hline & Telas 5 e 6 & Telas de Conteúdo: Definição sobre câncer & 100,0 \\
\hline & Tela 7 & Crescimento Tecidual & 100,0 \\
\hline & Tela 8 & Oncogêneses & 100,0 \\
\hline & Tela 9 & Imunologia Tumoral & 100,0 \\
\hline & Telas 10 e 11 & Classificação / Comportamento biológico & 100,0 \\
\hline & Telas 12 e 13 & Nomenclatura & 100,0 \\
\hline \multirow{2}{*}{$\begin{array}{l}\text { Módulo II } \\
\text { Epidemiologia do Câncer do Colo do Útero no Brasil e no mundo }\end{array}$} & Telas 14 e 15 & Epidemiologia do Câncer & 100,0 \\
\hline & Telas 16 e 17 & Epidemiologia do Câncer do Colo do Útero no Brasil e no mundo & 100,0 \\
\hline $\begin{array}{l}\text { Módulo III } \\
\text { Fatores de Risco do Câncer do Colo do Útero }\end{array}$ & Telas 18 e 19 & Fatores de Risco & 95,2 \\
\hline \multirow{2}{*}{$\begin{array}{l}\text { Módulo IV } \\
\text { Prevenção do Câncer do Colo do Útero e Autoexame da genitália } \\
\text { feminina }\end{array}$} & Telas 20 e 21 & Prevenção do câncer do colo do útero & 100,0 \\
\hline & Telas 22 e 23 & Autoexame da genitália feminina & 95,2 \\
\hline \multirow{21}{*}{$\begin{array}{l}\text { Módulo V } \\
\text { Controle do Câncer do Colo do Útero e o Exame de Papanicolau }\end{array}$} & Telas 24 e 25 & Controle do Câncer do Colo do Útero & 100,0 \\
\hline & Tela 26 & Exame do Papanicolau: Histórico & 100,0 \\
\hline & Telas 27 & Objetivo & 100,0 \\
\hline & Telas 28, 29 e 30 & Materiais Utilizados / Tipos de Espéculos & 100,0 \\
\hline & Telas 31 e 32 & Fixação do material à lâmina com spray e álcool / Recomendações & 95,2 \\
\hline & Tela 33 & Espátulas/Escovas & 100,0 \\
\hline & Tela 34 & Preenchimento do formulário do SISCAN / Identificação das lâminas & 95,2 \\
\hline & Telas 35 e 36 & Técnica do exame & 95,2 \\
\hline & Telas 37,38 e 39 & Coleta ectocervical / Coleta endocervical / Teste de Schiller & 100,0 \\
\hline & Telas 40 e 41 & Fixação do material / Recomendações & 95,2 \\
\hline & Telas 42 e 43 & Leitura e interpretação do exame & 90,5 \\
\hline & Telas 44 e 45 & $\begin{array}{l}\text { Principais achados no exame do Papanicolau: Amostra insatisfatória para avaliação / Amostra } \\
\text { satisfatória para avaliação / Recomendações }\end{array}$ & 95,2 \\
\hline & Telas 46 e 47 & $\begin{array}{l}\text { Situações especiais: Gestantes / Recomendações / Pós-menopausa / Recomendações / } \\
\text { Histerectomizadas / Recomendação }\end{array}$ & 95,2 \\
\hline & Telas 48 e 49 & $\begin{array}{l}\text { Resultado Normal / Dentro dos limites da normalidade no material examinado / } \\
\text { Recomendações / Alteraçõos celulares benignas (reativas ou reparativas) } \\
\text { Inflamação sem identificação de agente / Recomendações }\end{array}$ & 100,0 \\
\hline & Telas 50, 51 e 52 & $\begin{array}{l}\text { Resultado indicando metaplasia escamosa imatura / Recomendações / Resultado indicando } \\
\text { reparação / Recomendações / Resultado indicando atrofia com inflamação / recomendações / } \\
\text { Resultado indicando radiaçãoo / Recomendações / Achados microbiológicos / Recomendações }\end{array}$ & 95,2 \\
\hline & $\begin{array}{l}\text { Telas } 53,54,55 \\
\text { e } 56\end{array}$ & $\begin{array}{l}\text { Situações Especiais / Atipias de significado indeterminado em células escamosas / Células } \\
\text { escamosas atípicas de significado indeterminado, possivelmente não neoplásicas / Recomendações }\end{array}$ & 90,5 \\
\hline & Telas 57 e 58 & Corrimento vaginal / Etiologia do corrimento vaginal & 100,0 \\
\hline & Telas 59 e 60 & Exame da genitália feminina interna e externa & 100,0 \\
\hline & Telas 61e 62 & Observação Genitoperianal & 100,0 \\
\hline & Telas 63 e 64 & Toque Vaginal ou Toque Combinado & 100,0 \\
\hline & Telas 65 & Registro de enfermagem em consulta ginecológica & 95,2 \\
\hline
\end{tabular}


$\mathrm{Na}$ etapa de construção foi utilizada a plataforma Fred, que possui fácil navegabilidade. $\mathrm{O}$ acesso ao OVA ocorre pelo site CuidarTech , após o usuário realizar um cadastro, gerando um login e senha. Para acessar é necessário optar na Página Inicial (Figura 1) pelo curso "Consulta de Enfermagem em Ginecologia: foco na coleta do preventivo". O usuário ao clicar é apresentada a tela com os sete módulos, conforme a figura 2 .

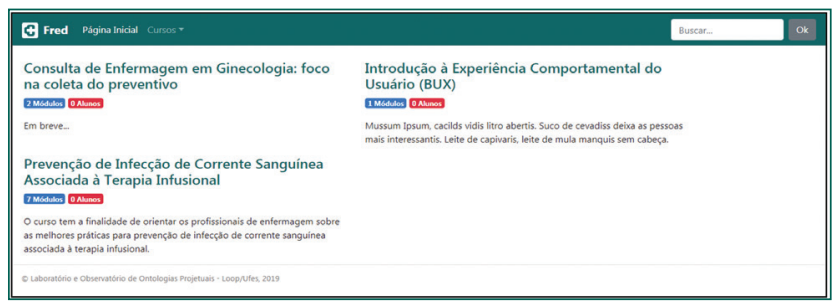

Figura 1. Página Inicial do OVA

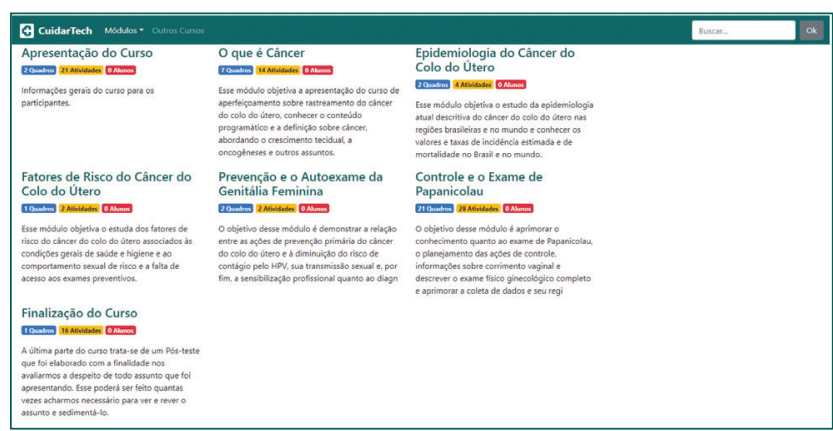

Figura 2. Tela de apresentação dos módulos do OVA

Ao clicar em "Apresentação do curso", o usuário tem acesso ao pré-teste (Figura 3). Após realizar o pré-teste pode-se acessar qualquer um dos cinco módulos de conteúdo conforme a necessidade de aprendizagem ou interesse do usuário. Ao final, no módulo de "Finalização do curso", estão incluídas as questóes de pós-teste. Os testes apresentam 26 questóes para avaliação do conhecimento.

Em relação a navegabilidade do OVA, conforme observa-se no módulo 1 - "O que é câncer" com o tópico "Definição sobre câncer” (Figura 4), após visualizar o conteúdo escrito e o infográfico, o estudante passará para a próxima tela, sendo necessário clicar no item "próximo", na parte superior das telas. Ao finalizar o módulo 1, o usuário pode acessar qualquer um dos outros módulos de conteúdo.

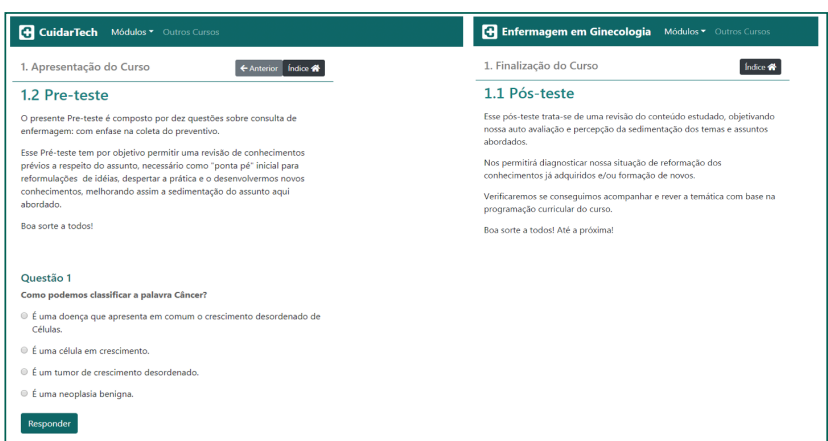

Figura 3. Telas "Apresentação e Finalização do Curso" com 0 pré e pós-teste

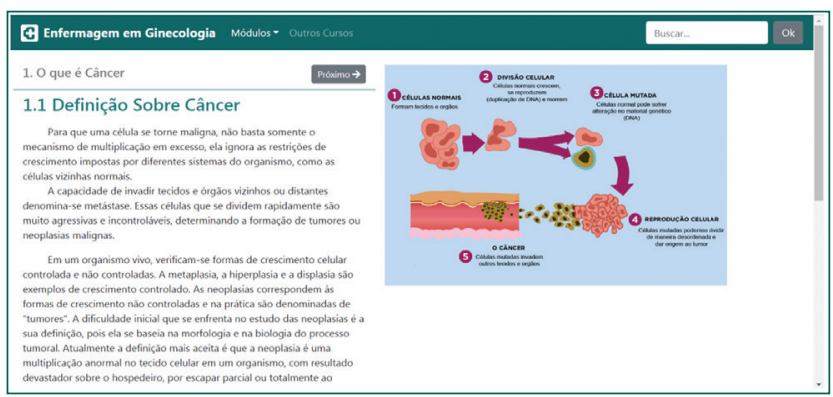

Figura 4. Tela "Definição sobre câncer" do OVA

\section{Discussão}

Esse OVA foi idealizado frente a um cenário mundial no qual o ensino a distância vem ganhando fortes evidências como método inovador, nos programas de educação continuada, que reconhece as dificuldades na disponibilidade de tempo dos profissionais de saúde. ${ }^{(16-18)}$ Propôs-se oferecer um produto inovador para o ensino em ginecologia, com a finalidade de atualizar os enfermeiros, com ênfase na importância da realização dos procedimentos de forma adequada, na leitura e interpretação do laudo do exame e determinação de condutas.

A coleta do exame de preventivo é considerado o meio mais eficaz para o diagnóstico do câncer de colo do útero, tendo em vista que a maioria dos casos ocorre de forma silenciosa, o que torna necessário atuar com competência, habilidade e acurácia na coleta do exame para rastreio. ${ }^{(19-21)}$

A adequabilidade da amostra do material está em grande parte, relacionada ao desempenho dos profissionais em realizarem a técnica de coleta, caracterizada basicamente pelo trabalho manual, que abrange desde a coleta até a emissão do laudo pelo 
laboratório de análise. A capacitação e a atualização da técnica permitem corrigir falhas nesse processo e consequentemente aumentar o número de lâminas satisfatórias, permitindo a detecção de lesôes pré-malignas precocemente, a trazer impacto positivo aos municípios que atendem ao programa de rastreamento do câncer do colo do útero. ${ }^{(22)}$

A escolha dos módulos do OVA levou em consideração a necessidade de atualização profissional, o aprimoramento de conhecimentos e a formação de um pensamento crítico para tomada de decisão com base nos resultados encontrados. Tendo em vista que o pensamento crítico é essencial para realização de uma prática clínica mais segura e eficiente. ${ }^{(20)}$

O uso de ilustraçóes, animaçóes, infográficos e áudio tornaram a hipermídia mais leve e efetiva para a compreensão, de acordo com as avaliaçôes e comentários dos juízes. A possibilidade de oferecer informaçôes de forma mais detalhada e atrativa, além da flexibilidade de uso, torna a hipermídia uma ferramenta dinâmica para o processo de ensino-aprendizagem. ${ }^{(7,17,18)}$

$\mathrm{Na}$ avaliação do conteúdo, os juízes destacaram como fatores importantes do OVA: os objetivos propostos de forma concisa, a maneira de apresentação do assunto, as imagens para a correta coleta do preventivo, a fidelidade do tema apresentado e a possibilidade de estimular o pensamento crítico nos profissionais de saúde. Todas estas questóes e a necessidade de capacitação continuada dos profissionais de saúde em diversas localidades dão indícios a vislumbrar o potencial de uso desse OVA como um novo espaço pedagógico. ${ }^{(20)}$

As tecnologias utilizadas para ensino a distância possibilitam o acesso à informaçôes, com facilidade e atualização da temática de forma rápida, para os profissionais de saúde, permitindo o acesso de vários locais, inclusive no local de trabalho. $\mathrm{O}$ funcionamento da plataforma escolhida permite ao profissional estudar e progredir no conteúdo em seu próprio ritmo e disponibilidade de tempo para estudo ${ }^{(14)}$. Fatores que denotam algumas características especiais de um OVA, importante à contribuição para o processo ensino-aprendizagem em saúde. ${ }^{(5,16,17)}$

Ainda, aponta-se a potencialidade deste OVA ser utilizado no ensino superior de enfermagem como uma ferramenta complementar no ensino-aprendizado desta tématica. As pesquisas de desenvolvimento e avaliação de tecnologias educacionais digitais para estudantes de graduação em enfermagem vem crescendo ao longo dos anos, apontando as vantagens de seu uso e abordam diferentes conteúdos como punção venosa periférica, assistência de enfermagem ao parto de risco habitual, doenças sexualmente transmissíveis, oxigenoterapia, processo de enfermagem, terapia intensiva, administração de medicamentos, sinais vitais, cateterismo vesical masculino e feminino, punção, heparinização de cateter totalmente implantado, entre outros. ${ }^{(4,5,8-10,16)}$

Da mesma forma que na educação permanente, as tecnologias educacionais podem ser incorporadas no ensino de enfermagem com o propósito de colaborar no desenvolvimento de competências e habilidades, apreensão de conteúdos inerentes à prática profissional, incentivando um processo de ensino-aprendizagem interativo, inovador e flexível. Por outro lado, entende-se que as tecnologias digitais aplicadas na graduação em enfermagem trazem desafios para docentes e discentes, no que se refere a organizar atividades específicas e com estrutura diferente das aplicadas nos espaços presenciais, o conteúdo deve ter uma sequência lógica, com uma abordagem autoexplicativa e dinâmica, as atividades precisam envolver e motivar os estudantes a aprenderem integrando suas experiências e conhecimentos prévios. ${ }^{(3-5,8-10,16-18)}$

Cabe ressaltar a importância do trabalho multidisciplinar na elaboração de tecnologias educacionais, assim, na construção e avaliação desse OVA esteve envolvida uma equipe de profissionais do design, programação, pedagogia, letras e linguística, enfermagem, entre outros. Dessa forma, no que tange a sua abordagem pedagógica, percebe-se o cuidado na elaboração de um material tecnológico com objetivos educacionais, com base em um alicerce pedagógico dinâmico e estruturado. ${ }^{(7,9,16,17)}$

\section{Contribuições para enfermagem, saúde ou política pública}

O OVA desenvolvido é um recurso tecnológico para colaborar na capacitação e atualização dos profissionais de saúde, em especial dos enfermeiros, quanto 
a técnica de coleta do exame preventivo do câncer do colo do útero e possibilitar a aquisição de novos conhecimentos e habilidades pelos acadêmicos de enfermagem.

\section{Conclusão}

O objeto virtual de aprendizagem para capacitação na prevenção e rastreamento do câncer do colo do útero possui sete módulos e 65 telas, com vídeos, infográficos, imagens e textos. A avaliação do conteúdo alcançou índice superior a $80 \%$ entre os juízes, apontando a qualidade e adequação do conteúdo. Assim, esta hipermídia é uma ferramenta viável e oportuna para ser utilizada na educaçáo e capacitação à distância. Como tecnologia educacional digital, o objeto virtual de aprendizagem poderá ser aplicado aos profissionais e acadêmicos de enfermagem, devido a sua inovação e relevância no contexto do rastreamento do câncer do colo do útero no país. Aponta-se como limitação a necessidade de estudos de avaliação da efetividade da tecnologia na aprendizagem dos diferentes usuários e contextos educacionais.

\section{Agradecimentos}

Agradecemos a equipe do Laboratório $\mathrm{LOOP}^{\oplus}$ (Laboratório e Observatório de Ontologias Projetuais), aos membros do CuidarTech ${ }^{\oplus}$ Laboratório de Tecnologias em Saúde, a profa. Dra. Janayna Casotti e a equipe do projeto Releitores, todos da Universidade Federal do Espírito Santo. Financiamento: Fundação de Amparo a Pesquisa do Espírito Santo (FAPES), processo número 80641440.

\section{Colaborações}

Davilla MSD, Primo CC, Almeida MVS, Leite FMC, Sant'Anna HC, Jensen R e Lima EFA declaram que contribuíram com a concepção do projeto, análise e interpretação dos dados, redação do artigo, revisão crítica relevante do conteúdo intelectual e aprovação final da versão a ser publicada.

\section{Referências}

1. Instituto Nacional de Câncer José Alencar Gomes da Silva (INCA). Estimativa 2020: incidência de câncer no Brasil. Brasília (DF): INCA; 2019. [citado 2020 Mar 31]. Disponível em: https://www.inca.gov.br/ publicacoes/livros/estimativa-2020-incidencia-de-cancer-no-brasil

2. Instituto Nacional de Câncer José Alencar Gomes da Silva (INCA). Colo de útero: detecção precoce. Brasília (DF): INCA; 2017. [citado 2018 Jun 07]. Disponível em: <http://www2.inca.gov.br/wps/wcm/connect/ tiposdecancer/site/home/colo_utero/deteccao_precoce>

3. Puggina CC, Amestoy SC, Fernandes HN, Carvalho LA, Báo AC, Alves F. Educação permanente em saúde: instrumento de transformação do trabalho de enfermeiros. Espaço Saúde. 2016;16(4):87-97.

4. Silveira MS, Cogo AL. The contributions of digital technologies in the teaching of nursing skills: an integrative review. Rev Gaúcha Enferm. 2017;38(2):e66204.

5. Luna IT, Pinheiro PN, Teixeira FO. Hypermedia for teaching nursing in a digital learning environment. Braz J Technol. 2018; 1(2):209-31.

6. Nietsche EA, Backes VM, Colomé CL, Ceratti RN, Ferraz F. Tecnologias educacionais, assistenciais e gerenciais: uma reflexão a partir da concepção dos docentes de enfermagem. Rev Lat Am Enfermagem 2005; 13(3):344-53

7. Antonio Junior W. Objetos virtuais de aprendizagem como recursos digitais educacionais. Pedag Foco. 2016;11(5):53-

8. Holanda VR, Pinheiro AK. Technology for education of sexually transmitted diseases: hypermedia validation Rev Enferm UFPE Online. 2016; 10(6):2082-90.

9. Frota NM, Galindo Neto NM, Barros LM, Pereira FG, Melo GA, Caetano $J A$. Hypermedia on peripheral venipuncture: effectiveness in teaching nursing students. Rev Bras Enferm. 2018;71(6):2983-9.

10. Oliveira LL, Mendes IC, Balsells MM, Bernardo EB, Castro RC, Aquino PS, et al. Educational hypermedia in nursing assistance at birth: building and validation of content and appearance. Rev Bras Enferm. 2019;72(6):1471-8.

11. Gil AC. Como elaborar projetos de pesquisa. 6a ed. São Paulo: Atlas; 2017

12. Marques JB, de Freitas D. The delphi method: characterization and potentialities for educational research. Pro-Posições. 2018;29(2):389.

13. Keller FS. "Good-bye, teacher...". J Appl Behav Anal. 1968;1(1):79-89.

14. Eyre HL. Keller's personalized system of instruction: was it a fleeting fancy or is there a revival on the horizon? Behav Anal Today. 2007;8(3):317-20.

15. Watson WR, Watson SL. Principles for personalized instruction. Instructional-design theories and models. In: Reigeluth CM, Beatty BJ, Myers Rd, editors. Instructional -design theories and models: the learner-centered paradigm of education. New York: Routledge; 2016. Vol. 4 Cap. 4, p. 94-102.

16. Salvador PT, Rodrigues CC, Ferreira Júnior MA, Fernandes MI, Martins JC, Santos VE. Construção de hipermídia para apoio ao ensino da sistematização da assistência de enfermagem. Rev Gaúcha Enferm. 2019;40:e20180035. 
17. Costa IK, Tibúrcio MP, Melo GS, Leite JE, Dantas RA, Torres GV. Construction and validation of a distance Basic Life Support Course. Rev Bras Enferm. 2018;71(Suppl 6):2698-705.

18. Vaona A, Banzi R, Kwag KH, Rigon G, Cereda D, Pecoraro V, et al. Elearning for health professionals. Cochrane Database of Systematic Reviews. 2018 Jan 21,1(1): CD011736.

19. Junior Ormonde JC, Oliveira LD, Sá RM. Fatores de adesão e não adesão das mulheres ao exame colpacitológico. Rev Eletrôn Gestão Saúde. 2015;6(1):184-200.
20. Ghezzi JF, Higa EF, Nalo DM, Biffe CR, Lemes MA, Marin MJ. Metodologias de aprendizagem ativa e a formação do enfermeiro com pensamento crítico: revisão integrativa da literatura. Investigação Qualitativa em Educação CIAIQ. 2019;1:478-87. [Atas do $8^{\circ}$ Congresso Ibero-Americano em Investigação Qualitativa].

21. Silva NS, Santos CB, Lotti RC. Conhecimento, Atitude e Prática do Exame Papanicolau. J Health Connections. 2018;6(5):28-42.

22. Damacena AM, Luz LL, Mattos IE. Cervical cancer screening in Teresina, Piauí, Brazil: evaluation study using data of the Cervical Cancer Information System, 2006-2013. Epidemiol Serv Saude. 2017;26(1):71-80. 